\title{
Hydration Water Dynamics in Undercooled Aqueous Solutions of Hydrophobic Ions
}

\author{
S. Bradl and E. W. Lang* \\ Institut für Biophysik und physikalische Biochemie, Universität Regensburg, \\ POB 101042, W-8400 Regensburg, FRG
}

Received: March 3, 1993; In Final Form: May 19, 1993

\begin{abstract}
Deuterium spin-lattice relaxation times $\left({ }^{2} \mathrm{H} T_{1}\right)$ are reported for the first time as a function of temperature $(180-300 \mathrm{~K})$, pressure $(p \leq 225 \mathrm{MPa})$, and composition $(c \leq 5 \mathrm{~m})$ in undercooled aqueous solutions of tetraalkylammonium ions $\left(\mathbf{R}_{4} \mathbf{N}^{+}\right)$. A comparison with related investigations of undercooled alkali-metal halide solutions allows the competing influence of coulombic, hydrophobic, and $\mathrm{H}$-bond interactions upon the dynamic structure of the random, transient $\mathrm{H}$-bond network to be studied. Solvent dynamics are seen to be closely related to the glass-forming tendency of undercooled tetramethylammonium $\left(\mathrm{Me}_{4} \mathrm{~N}^{+}\right)$and tetrapropylammonium $\left(\mathrm{Pr}_{4} \mathrm{~N}^{+}\right)$bromide solutions and to the clathrate-forming tendency of undercooled tetrabutylammonium $\left(\mathrm{Bu}_{4} \mathrm{~N}^{+}\right)$ bromide solutions.
\end{abstract}

\section{Introduction}

Nuclear magnetic resonance studies provide valuable information about rotational and translational diffusion of water molecules and ions in undercooled electrolytes. ${ }^{1,2}$ In metastable water at low temperatures, hydrogen-bonding interactions dominate the dynamic structure of the random, transient $\mathrm{H}$-bond network. ${ }^{3}$ Hence, molecular motions become highly cooperative close to the low-temperature limit of the metastable phase. 3,4 Dissolved ions disturb the local structure of the H-bond network and alter molecular motions of coordinated water molecules in a characteristic fashion. Molecular ions with apolar groups like tetraalkylammonium $\left(\mathbf{R}_{\mathbf{4}} \mathbf{N}^{+}\right)$ions allow the competing influence of the Coulomb effect of the charge density and the hydrophobic effect of the apolar surface on the network to be studied. ${ }^{5}$ Hence, aqueous solutions of $\mathbf{R}_{\mathbf{4}} \mathrm{N}^{+}$ions were long considered to provide model systems for a study of hydrophobic hydration and hydrophobic interaction phenomena. Though the concept of hydrophobic hydration is widely used in chemistry and biology to describe the reduction in entropy upon solvation of apolar solutes in water, its molecular basis is not well understood yet. ${ }^{6,7}$ Despite many thermodynamic data, only very few investigations of the molecular dynamics in these systems exist, ${ }^{6,7}$ and direct structural studies were reported only recently. ${ }^{8-10}$

Thermodynamic and spectroscopic results suggest that the solvation of apolar groups (hydrophobic hydration) increases the amount of order in the random, transient $\mathrm{H}$-bond network of the coordinated water molecules and reduces their mobility. Symmetric tetraalkylammonium ions may be considered model systems to study the competing influence of coulombic, hydrophobic, and $\mathrm{H}$-bonding interactions upon the dynamic structure of the $\mathrm{H}$-bond network of water. The structure-enhancing effect of the inert alkyl chains is expected to dominate if the latter are long enough. According to all measures of "structure-making", $\mathrm{Me}_{4} \mathrm{~N}^{+}$ions are considered as structure breakers and $\operatorname{Pr}_{4} \mathrm{~N}^{+}$and $\mathrm{Bu}_{4} \mathrm{~N}^{+}$ions as structure makers in the sense of more and stronger $\mathrm{H}$-bonds between the coordinated water molecules. ${ }^{6,7}$ The hydration structures involved may resemble clathratelike cages which would be favored by the network organization of liquid water. ${ }^{11,12}$ Any such structures seem to be predicted by computer simulations ${ }^{13-15}$ but could not yet be detected in neutron scattering experiments on aqueous tetramethylammonium $\left(\mathrm{Me}_{4} \mathrm{~N}^{+}\right)$halides.,9

Valuable new information may be obtained in undercooled aqueous $\mathbf{R}_{4} \mathbf{N}^{+}$solutions. Because of reduced thermal excitations, hydrophobic hydration structures would be more stable and longlived and hence would cause a dramatic slowing down of orientational and positional fluctuations of water molecules.
Except for calorimetric studies of the glass transition in these systems $^{16-18}$ and DTA and ionic conductivity measurements in undercooled tetrabutylammonium bromide ( $\mathrm{Bu}_{4} \mathrm{NBr}$ ) solutions, ${ }^{19}$ virtually no further investigations of the physical properties of aqueous solutions of $\mathbf{R}_{\mathbf{4}} \mathrm{N}^{+}$ions at low temperatures exist despite the long known peculiarity that these solutes also suppress the temperature of maximum density of water. ${ }^{6}$

We report in this study deuterium $\left({ }^{2} \mathrm{H}\right)$ relaxation time $\left(T_{1}\right)$ measurements in undercooled aqueous tetramethylammonium $\left(\mathrm{Me}_{4} \mathrm{~N}^{+}\right)$, tetrapropylammonium $\left(\mathrm{Pr}_{4} \mathrm{~N}^{+}\right)$, and tetrabutylammonium $\left(\mathrm{Bu}_{4} \mathrm{~N}^{+}\right)$bromide solutions. To probe many different states of the hydrogen-bond network, temperature $(T)$, pressure $(p)$, and concentration $(c)$ have been varied over a large range.

\section{Experiment}

Tetramethylammonium bromide $\left(\mathrm{Me}_{4} \mathrm{NBr}\right)$, tetrapropylammonium bromide $\left(\mathrm{Pr}_{4} \mathrm{NBr}\right)$, and tetrabutylammonium bromide $\left(\mathrm{Bu}_{4} \mathrm{NBr}\right)$ were obtained from Aldrich Chemicals (Steinheim, FRG), and heavy water $\left(D_{2} \mathrm{O}, 99.8\right.$ atom \%) was purchased from Merck (Darmstadt, FRG). The salts were dried on a vacuum line for $24 \mathrm{~h}$, and the solutions were prepared by weighing the proper amount of salt to $3 \mathrm{~mL}$ of heavy water. Afterward, the solutions were degassed on a vacuum line by at least five freezepump-thaw cycles and then filled into the high-pressure glass capillary as described elsewhere. ${ }^{2}$ To reach high degrees of undercooling emulsions had to be used, however. As the supporting alkane phase, deuterated methylcyclohexane (MCH$d_{14}$, Riedel-deHaen, Seelze, FRG) and a small amount of the surfactant sorbitan tristearate (Span 65, Merck, Darmstadt, FRG) were used. Emulsions were then produced under an argon atmosphere in a glovebag (AtmosBag, Aldrich Chemicals, Steinheim, FRG) by pressing the alkane/aqueous solution mixture through a stainless steel net (Spörl \& Co., Sigmaringendorf, FRG) within a syringe. Afterward, the emulsion had to be filled into the bigh-pressure capillaries on the vacuum line.

All experiments were performed on a Bruker MSL 300 multipurpose solid-liquid NMR spectrometer equipped with a variable temperature unit VT 100 (Bruker, Rheinstetten, FRG) and a pulsed magnetic field gradient unit BZ 18B. The gradient unit was modified for a digital setting of the gradient amplitude by a series of fixed resistances. The probe for the $T_{1}$ measurements consisted of a home-built $10-\mathrm{mm}$ probehead with an external ${ }^{19} \mathrm{~F}$ lock suitable for operation in the range $44-47 \mathrm{MHz}$. The probe for self-diffusion measurements consisted of a homo-built 10$\mathrm{mm}$ probehead with two Helmholz coils suitable for operation in the range $280-300 \mathrm{MHz}$. Details of the probe design have been 
described elsewhere. ${ }^{2,53}$ The design assumes that the gradient coils are thermally isolated from the sample space so the coils remain at constant temperature during variable-temperature measurements.

Spin-lattice relaxation times $T_{1}$ were determined with the inversion-recovery pulse sequence $(\pi-\tau-\pi / 2)$ with alternating phases. Data were analyzed with a three-parameter nonlinear least-squares regression program. The relaxation times are considered reliable to $\pm 10 \%$.

For the self-diffusion measurements the Fourier transform pulsed gradient spin echo method (FT-PGSE) was applied to the proton magnetization. The decay of the spin echo amplitude was then monitored as a function of the field gradient strength $g=$ $k I$ with $I$ the gradient coil current which was increased digitally for 10-15 different settings. The applied gradient strength then typically ranged from $9.6 \times 10^{-3}$ to $37.1 \times 10^{-3} \mathrm{~T} / \mathrm{m}$. The time scale during which molecular diffusion is monitored in the PGSE experiment ranged from 50 to $70 \mathrm{~ms}$. The standard deviation of the fitted diffusion coefficients was always better than $2 \%$. Reproducibility considerations, however, indicate the overall accuracy is $\pm 5 \%$.

The temperature was varied with a flow of cold nitrogen gas appropriately heated and regulated by the VT 100 unit. It was measured at the center of the rf coil with a chromel-alumel thermocouple (Philips, Kassel, FRG) before and after each series of inversion-recovery experiments. The temperatures are reliable to $\pm 1 \mathrm{~K}$. The pressure was generated with a home-built highpressure apparatus suitable for operation up to $400 \mathrm{MPa}$ and was measured with a Bourdon gauge (Heise, Connecticut) with a precision of $\pm 0.5 \mathrm{MPa}$.

\section{Theory}

The relaxation of the deuterium $\left({ }^{2} \mathrm{H}\right)$ nuclei is dominated by a coupling of the electric quadrupole moment $e Q$ of the nucleus to the electric field gradient (efg) eq at the nucleus due to the surrounding electronic and nuclear charge distributions. In water the efg at the deuterium nucleus originates almost exclusively from intramolecular charge distributions. The relevant interaction Hamiltonian is given in a laboratory-fixed frame $(L)$ by ${ }^{20}$

$$
H_{Q}(t)=e Q / 2 I(I-1) \hbar \sum(-1)^{m} T_{2 m}^{\mathrm{L}} R_{2-m}^{\mathrm{L}}(t)
$$

$I$ is the spin quantum number $\left({ }^{2} \mathrm{H}, I=1\right)$. The $T_{2 m}$ are secondrank irreducible tensor operators, and the $R_{2 m}$ are components of the second-rank irreducible field gradient tensor. The latter are most conveniently expressed in a molecule-fixed principal axis system ( $P)$, where they become constants. Conventionally, the efg tensor is characterized by the quadrupole coupling constant (QCC)

$$
\chi=e^{2} Q q_{z z}^{P} / h
$$

and by the asymmetry factor

$$
\eta=\left(q_{x x}-q_{y y}\right) / q_{z z}
$$

The efg at the deuterium nucleus in the water molecule is nearly axially symmetric; hence $\eta=0$ to good approximation.

The transformation from the molecule-fixed to the laboratoryfixed (L) frame can be effected with the aid of second-rank Wigner rotation matrices. ${ }^{21}$ It depends on the motional model used to represent the orientational fluctuations of the water molecules. The latter modulate the interaction and render the transformation time dependent.

In the random, transient network model of liquid water orientational fluctuations of water molecules may be divided into fast oscillatory quasi-lattice vibrations (QLV) and slower diffusional rearrangements of the network topology.22 The shorttime reorientations consist of fast librations about an equilibrium orientation in the quasi-static network. On a longer time scale this equilibrium orientation will change due to slower rotational diffusive motions, thereby leading to a permanent rearrangement of the network topology.

Except with extreme undercooling, the molecular motions in water are fast compared to currently available NMR frequencies $\left(\omega / 2 \pi<10^{9} \mathrm{~Hz}\right)$. The quadrupole relaxation of the ${ }^{2} \mathrm{H}$ nuclear spins then proceeds exponentially at a frequency-independent rate given by ${ }^{20}$

$$
\begin{array}{r}
R_{1}=R_{2}=3 \pi^{2}(2 I+3) / 2 I^{2}(2 I-1)(e Q / h)^{2} \times \\
\qquad\left\langle R_{20}(\tau) R_{20}(0)\right\rangle \mathrm{d} \tau
\end{array}
$$

The integral over the efg time-correlation function $G(\tau)$

$$
G_{20}(\tau)=\left\langle R_{20}(\tau) R_{20}(0)\right\rangle
$$

defines an average correlation time $\langle\tau\rangle$

$$
\langle\tau\rangle=\int G_{20}(\tau) / G_{20}(0) \mathrm{d} \tau
$$

which provides a time scale for those molecular motions that can be studied with NMR. In liquid water the slow diffusional tumbling modes are isotropic to good approximation, ${ }^{23-26}$ hence

$$
G_{20}(t)=(1 / 5)\langle e q\rangle_{\mathrm{QLV}}^{2} \exp \left(-t / \tau_{\mathrm{or}}\right)
$$

yielding in the fast-motions limit

$$
R_{1}=\left(3 \pi^{2} / 2\right)\langle\chi\rangle_{\mathrm{QLV}}^{2} \tau_{\text {or }}
$$

The effect of the fast librations is seen to reduce the QCC to a librationally averaged value of

$$
\langle\chi\rangle_{\mathrm{QLV}}=\langle e q\rangle e Q / h=\left(e q_{z z}^{\mathrm{P}} e Q / h\right) \Gamma=\chi \Gamma
$$

The motional averaging factor $\Gamma$ is given by

$\Gamma^{2}=\sum\left|\left\langle D_{0 m}(\Omega)\right\rangle+\eta / \sqrt{ } 6\left[\left\langle D_{-2 m}(\Omega)\right\rangle+\left\langle D_{2 m}(\Omega)\right\rangle\right]\right|^{2}$

where $D_{m m^{\prime}}(\Omega)$ are elements of the second-rank Wigner rotation matrix and $\Omega$ denotes the Euler angles that transform from the molecule-fixed efg principal frame to the librationally averaged molecular frame. ${ }^{24,27}$

The deuterium spin $\left({ }^{2} \mathrm{H}, I=1\right)$ relaxation proceeds exponentially even in the slow-motions regime $\left(\omega \tau_{\text {or }}>1\right)$. The spinlattice relaxation rate $R_{1}=1 / T_{1}$ may then be expressed as ${ }^{20}$

$$
R_{1}\left({ }^{2} \mathrm{H}\right)=\left(3 \pi^{2} / 2\right)\langle\chi\rangle_{\mathrm{QLV}}^{2} J\left(\omega, \tau_{\mathrm{or}}\right)
$$

with

$$
\begin{gathered}
J\left(\omega \tau_{\mathrm{or}}\right)=\sum m^{2} g_{2 m}\left(m \omega \tau_{\mathrm{or}}\right) \\
g_{2 m}(m \omega)=\int G_{2 m}(\tau) \exp \left(i m \omega \tau_{\mathrm{or}}\right) \mathrm{d} \tau \\
=G_{2}(0) \tau_{\mathrm{or}} /\left[1+\left(m \omega \tau_{\mathrm{or}}\right)^{2}\right]
\end{gathered}
$$

In complex systems such as the solutions considered in this study, the water molecules may experience different environments with different relaxation characteristics due to more or less constrained motional modes. A simple two-mode approximation ${ }^{28-30}$ of the hydration water dynamics in terms of an isotropic overall tumbling mode and a spatially restricted, hence anisotropic, local mode may prove adequate to represent the orientational fluctuations of the water molecules coordinated to the apolar ions. Then one obtains instead of eq 13

$$
g_{2 m}(m \omega)=S^{2} \tau_{\text {or }} /\left(1+\left(m \omega_{\mathrm{L}} \tau_{\text {or }}\right)^{2}\right)+\left(1-S^{2}\right) \tau /\left(1+\left(m \omega_{\mathrm{L}} \tau\right)^{2}\right)
$$

with the generalized order parameter $S^{2}$ providing a measure of the spatial restriction of the local mode ${ }^{29}$ and $1 / \tau=1 / \tau_{\text {or }}+1 / \tau_{f}$. In case of time scale separation $\left(\tau=\tau_{f} \ll \tau_{o r}\right)$, it gives the amount of averaging of the static spin-lattice coupling by the anisotropic fast mode. ${ }^{28}$ 
Another alternative is to resort to empirical correlation functions often used to represent the relaxation in complex systems in a certain time window. A parallel relaxation of nonequilibrium states leads to a distribution of correlation times spreading from fast anisotropic local modes to slow isotropic tumbling modes. Experimental relaxation rate curves dictate an asymmetric distribution conveniently represented by the Cole-Davidson form ${ }^{31}$

$$
\begin{gathered}
G(t / \tau)=\int G(0) \exp (-t / \tau) p(\tau) \mathrm{d} \tau \\
p(\tau)= \begin{cases}\pi^{-1} \sin (\beta \pi)\left[\tau /\left(\tau_{0}-\tau\right)\right]^{\beta} & 0<\tau<\tau_{0} \\
0 & \tau_{0}<\tau\end{cases}
\end{gathered}
$$

The mean correlation time is given by the first moment of the distribution $p(\tau)$

$$
\langle\tau\rangle=\int \tau p(\tau) \mathrm{d} \tau=\beta \tau_{0}
$$

$\beta$ characterizes the width of the distribution, and $\tau_{0}$ marks the low-frequency cut off beyond which the modes are too slow to contribute to the relaxation. The asymmetric CD distribution is thus a convenient means to account for high-frequency motions contributing to the relaxation.

The corresponding density of states function is again obtained via a Fourier-Laplace transform of the correlation function and reads

$$
g(\omega \tau)=\sin \left[\beta \arctan \left(\omega \tau_{0}\right)\right] / \omega\left[1+\left(\omega \tau_{0}\right)^{2}\right]^{\beta / 2}
$$

After insertion of the appropriate spectral density function $g\left(\omega_{L} \tau\right)$ into eqs 11 and 12 , the corresponding relaxation rates are obtained.

\section{Results}

Aqueous Tetramethylammonium $\left(\mathrm{Me}_{4} \mathrm{~N}^{+}\right)$Bromide Solutions: $\left(\mathrm{CH}_{3}\right)_{4} \mathrm{NBr} / \mathrm{D}_{2}$ O. Deuterium spin-lattice relaxation times $T_{1^{-}}$ $\left({ }^{2} \mathrm{H}\right)$ have been measured in undercooled solutions of TMABr in heavy water at the concentrations $c=1,2$, and $3 m$ corresponding to compositions $R=$ (moles of $\left.D_{2} \mathrm{O}\right) /$ (mole of salt) $=50,25$, and 17 , respectively. The $2 m$ solution corresponds to a composition where there is just enough water to hydrate the $\mathrm{Me}_{4} \mathrm{~N}^{+}$cations according to an estimated coordination number $N_{\mathrm{h}} \approx 25.32$ The pressure has been varied from $p=0.1$ to $225 \mathrm{MPa}$, and the temperature has been changed from 350 down to $200 \mathrm{~K}$.

Within the range of pressures applied only a small increase of the rotational mobility $\left(T_{1}\right)$ of the water molecules with density could be observed for the concentrations investigated (Figure 1). With increasing concentration the ${ }^{2} \mathrm{H} T_{1}$ decrease at high temperatures ( $T>240 \mathrm{~K}$ ) compared to those of pure water. At low temperatures $(T<240 \mathrm{~K})$ the relaxation time curves cross the corresponding $T_{1}(\mathrm{~T})$ curve of pure water (Figure 2). At a given low temperature $T_{1}\left({ }^{2} \mathrm{H}\right)$ is longer the higher the concentration of dissolved $\mathrm{Me}_{4} \mathrm{~N}^{+}$cations is. As almost no density dependence of $T_{1}$ has been observed, this observation holds at all pressures applied. This behavior of the ${ }^{2} \mathrm{H} T_{1}$ in $\mathrm{Me}_{4} \mathrm{~N}^{+} / \mathrm{D}_{2} \mathrm{O}$ solutions is quite different from the observations made in undercooled alkali metal and alkali earth halide solutions investigated in the same pressure range. ${ }^{1,2}$ At ambient conditions ( $300 \mathrm{~K}, 0.1 \mathrm{MPa})$, the ${ }^{2} \mathrm{H} T_{1}$ in these simple electrolyte solutions are either shorter or longer than the corresponding pure water results depending on the charge density of the cation. The same behavior is found also for all simple electrolyte solutions under high hydrostatic pressure $(p=225 \mathrm{MPa})$ in the whole temperature range studied. Hence, it is characteristic for a H-bond network, which is strongly disturbed by either large thermal excitations or high hydrostatic pressure. It is, however, quite different in undercooled simple electrolyte solutions at ambient pressure where the ${ }^{2} \mathrm{H} T_{1}$ are always longer than the pure water results independent of the charge density of the cations. All dissolved atomic ions thus disrupt the $\mathrm{H}$-bond network at low temperatures and cause the solvent mobility to increase. It is remarkable that
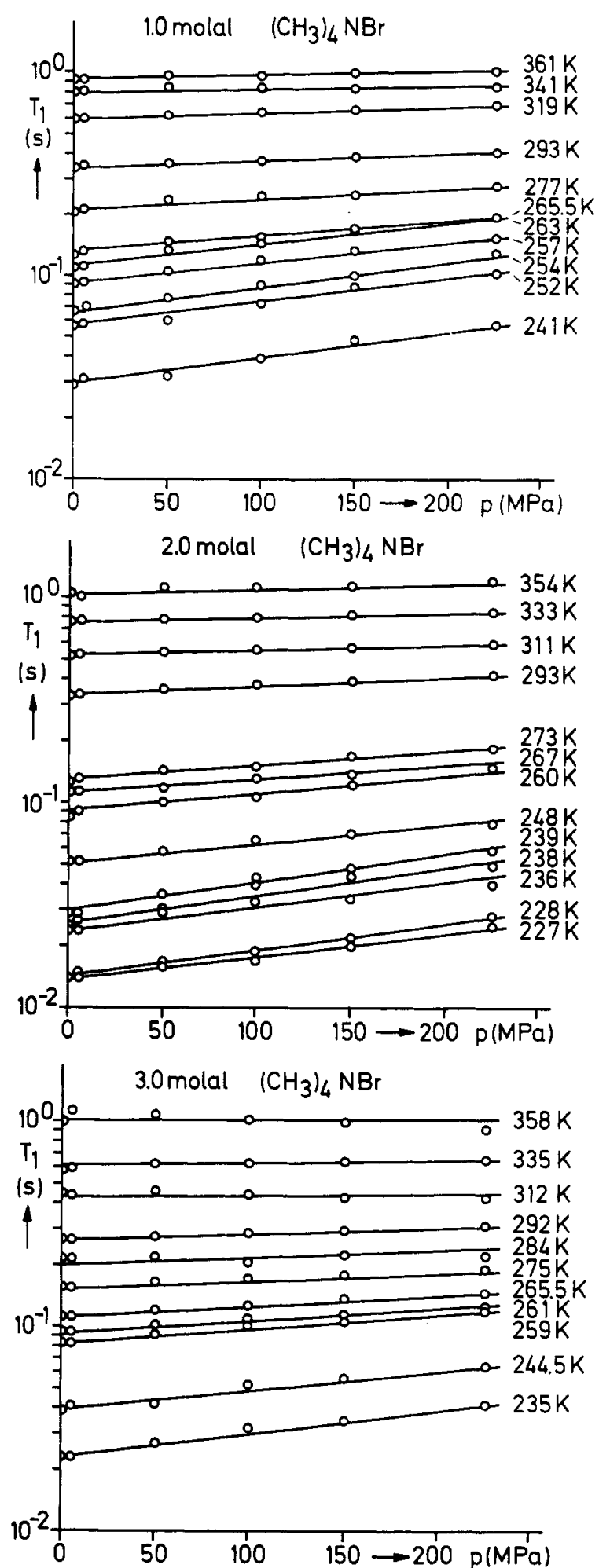

Figure 1. Isothermal pressure dependence of the water deuteron $T_{1}$ in aqueous tetramethylammonium bromide solutions at the concentrations 1,2 , and $3 \mathrm{~m}$. Concentrations are given on a molality scale. Full lines are drawn to guide the eye.

the latter behavior is observed in undercooled $\mathrm{TMA}^{+} / \mathrm{D}_{2} \mathrm{O}$ solutions even under high hydrostatic pressure.

Aqueous Tetrapropylammonium $\left(\mathrm{Pr}_{4} \mathrm{~N}^{+}\right.$) Bromide Solutions: $\left(\mathrm{C}_{3} \mathrm{H}_{7}\right)_{4} \mathrm{NBr} / \mathrm{D}_{2} \mathrm{O}$. The deuterium spin-lattice relaxation has been studied in $\operatorname{Pr}_{4} \mathrm{NBr} / \mathrm{D}_{2} \mathrm{O}$ solutions at the concentrations $c=$ $0.3,1.4,3$, and $5 m$ corresponding to compositions $R=167,36$, 17 , and 10 , respectively. The $1.4 \mathrm{~m}$ solution contains the proper amount of water to fully hydrate the $\mathrm{Pr}_{4} \mathrm{~N}^{+}$cations according to an estimated coordination number $N_{\mathrm{h}}=36.32$ Pressure and temperature have been varied over the same range as in case of the $\mathrm{Me}_{4} \mathrm{NBr} / \mathrm{D}_{2} \mathrm{O}$ solutions. Again, no strong dependence of the ${ }^{2} \mathrm{H} T_{1}$ on density could be detected. 


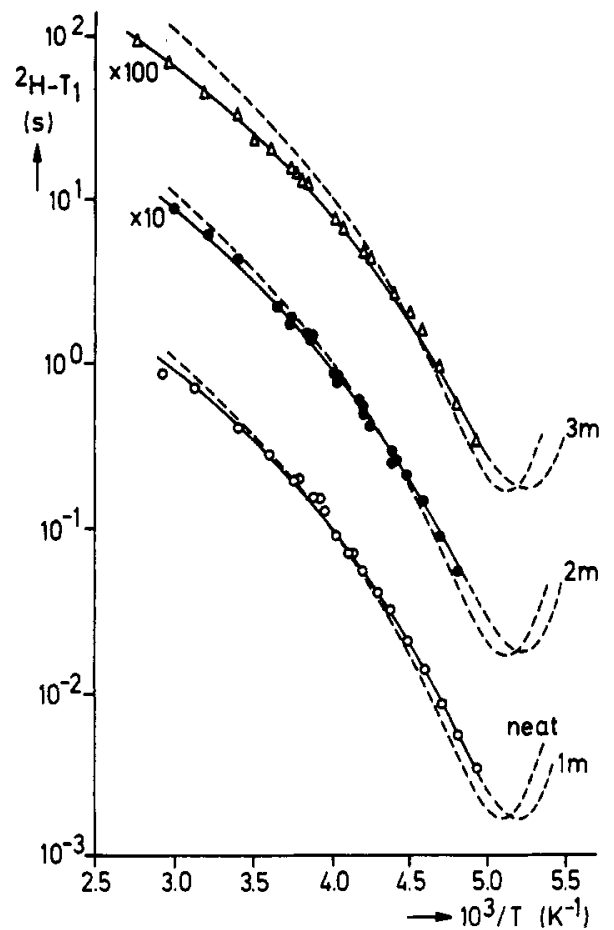

Figure 2. Isobaric $(p=225 \mathrm{MPa})$ temperature dependence of the water ${ }^{2} \mathrm{H} T_{1}$ of undercooled aqueous tetramethylammonium bromide solutions at the concentrations indicated. Full lines correspond to relaxation times calculated with the $C D$ distribution of correlation times model as discussed in the text. The broken line represents the corresponding relaxation times of neat $\mathrm{D}_{2} \mathrm{O} .^{2}$

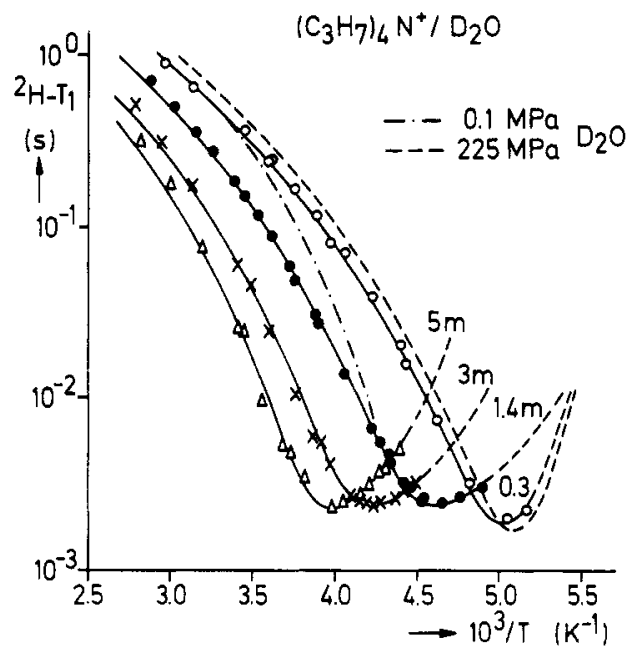

Figure 3. Isobaric $(p=225 \mathrm{MPa})$ temperature dependence of the water ${ }^{2} \mathrm{H} T_{1}$ of undercooled aqueous tetrapropylammonium bromide solutions at the concentrations indicated. Full lines correspond to the $C D$ distribution of correlation times model as discussed in the text. The broken lines represent corresponding relaxation times of pure $\mathrm{D}_{2} \mathrm{O}$ at $p$ $=225 \mathrm{MPa}^{2}$ and $p=0.1 \mathrm{MPa}^{54}$

Under high pressure dissolved $\mathrm{Pr}_{4} \mathrm{~N}^{+}$cations reduce the ${ }^{2} \mathrm{H} T_{1}$ of the water with increasing concentration at all temperatures investigated (Figure 3). At normal pressure this is true also except for the most dilute $(c=0.3 \mathrm{~m})$ solution where the average rotational mobility is increased considerably at low temperatures. For all concentrations the isobaric relaxation time curves $T_{1}(T)$ exhibit a minimum which shifts to higher temperatures with an increasing amount of dissolved ions. This positive temperature shift of $T_{\min }$ in $\mathrm{Pr}_{4} \mathrm{~N}^{+}$solutions is different from $\mathrm{Me}_{4} \mathrm{~N}^{+}$solutions where $T_{\min }$ seems to shift to lower temperatures with concentration (Figure 4). Also, the relaxation time at the minimum becomes longer with increasing concentration for solution compositions $R$ $\geq 36$ and remains roughly constant in solutions with $R<36$. Hence, the relaxation efficiency is reduced in the hydration water compared to bulk water. The shape of the relaxation time curves

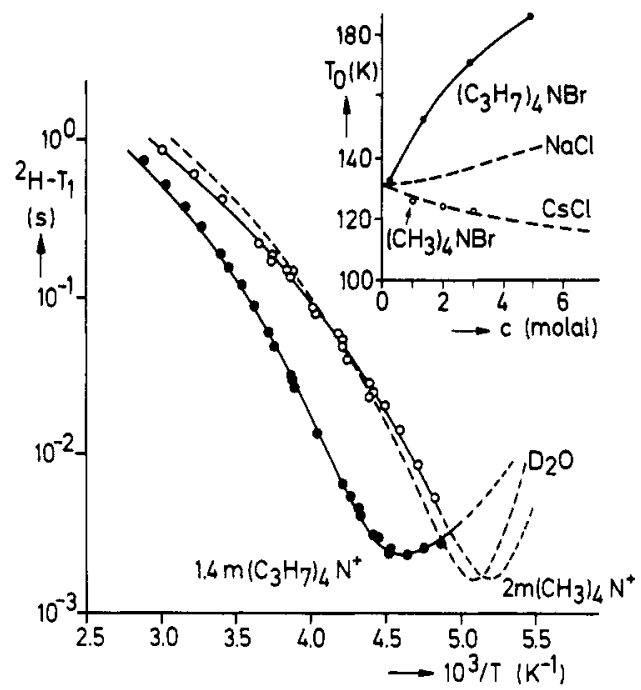

Figure 4. Isobaric ( $p=225 \mathrm{MPa})$ temperature dependence of the hydration water ${ }^{2} H T_{1}$ of undercooled aqueous solutions of tetrapropylammonium bromide and tetramethylammonium bromide. The broken line corresponds to pure $\mathrm{D}_{2} \mathrm{O}^{2}$ The insert gives the concentration dependence of the ideal glass transition temperature $T_{0}(R)=T_{\min }(R)$ $-66 \mathrm{~K}$ of these solutions with $T_{\min }$ corresponding to the temperature of the $T_{1}$ minimum. Note that the latter was not obtained by direct measurement in the tetramethylammonium bromide solutions.

is asymmetric around the broad minimum with a less pronounced temperature dependence on the low temperature side $\left(T<T_{\min }\right)$. Otherwise their shape does not seem to change much in the concentration range $c=1.4-5 \mathrm{~m}$. Similar observations have been made for the high-pressure relaxation rate curves of undercooled aqueous solutions of strongly hydrating atomic ions. ${ }^{33-37}$

Aqueous Tetrabutylammonium $\left(\mathrm{Bu}_{4} \mathrm{~N}^{+}\right)$Bromide Solutions: $\left(\mathrm{C}_{4} \mathrm{H}_{9}\right)_{4} \mathrm{NBr} / \mathrm{D}_{2} \mathrm{O}$. The deuterium spin-lattice relaxation has been studied in $\mathrm{Bu}_{4} \mathrm{NBr} / \mathrm{D}_{2} \mathrm{O}$ solutions at the concentrations $c$ $=0.3,1.2$, and $3 m$ corresponding to compositions $R=167,42$, and 17 , respectively. According to published coordination number estimates $N_{\mathrm{b}}=42,32$ the $1.2 \mathrm{~m}$ solution provides just enough water molecules to fully hydrate the cations. Contrary to the $\mathrm{Me}_{4} \mathrm{~N}^{+}$and $\mathrm{Pr}_{4} \mathrm{~N}^{+}$solutions a positive pressure effect upon the ${ }^{2} \mathrm{H} T_{1}$ could be observed in the $0.3 \mathrm{~m}$ solutions, the $1.2 \mathrm{~m}$ solution showed no pressure dependence at all, and the $3 \mathrm{~m}$ solution exhibited a strong negative pressure dependence on the ${ }^{2} \mathrm{H} T_{1}$.

With decreasing temperature, the ${ }^{2} \mathrm{H} T_{1}$ in $\mathrm{Bu} 4 \mathrm{NBr} / \mathrm{D}_{2} \mathrm{O}$ solutions show a complex behavior, which must be related to the complex phase diagram of these solutions. ${ }^{19}$ They are not glass forming for any concentration ${ }^{18}$ but instead form clathrate hydrates with the formula $\mathrm{Bu}_{4} \mathrm{~N}^{+} \mathrm{Br}-32.8 \mathrm{H}_{2} \mathrm{O}^{38}$ The $1.2 \mathrm{~m}$ solution is thus close to the eutectic composition $(R=32.8)$ of the corresponding clathrate hydrate. The respective melting $\left(T_{\mathrm{MC}}\right)$ and homogeneous nucleation $\left(T_{\mathrm{HC}}\right)$ temperature of the corresponding clathrate hydrate as well as the homogeneous nucleation temperature of ice $\left(T_{\mathrm{HI}}\right)$ are indicated in Figure 5 , which compares the temperature dependence of the water ${ }^{2} \mathrm{H} T_{1}$ in the investigated solutions at normal $(0.1 \mathrm{MPa})$ and high $(225$ $\mathrm{MPa}$ ) pressure. The relaxation times decrease with increasing concentration with the strongest temperature dependence found for the highest concentration. On approaching the homogeneous nucleation temperature $T_{\mathrm{HC}}$, the relaxation times level off and become independent of temperature until they merge with the corresponding relaxation time curve measured in pure heavy water under high pressure (225 MPa). At temperatures $T<T_{\mathrm{HI}}$ the ${ }^{2} \mathrm{H} T_{1}$ become indistinguishable from those of pure heavy water down to the lowest temperatures. ${ }^{2,24}$ At the highest concentration the ${ }^{2} \mathrm{H} T_{1}$ could not be obtained below $T_{\mathrm{HC}}$ because of too broad lines. 


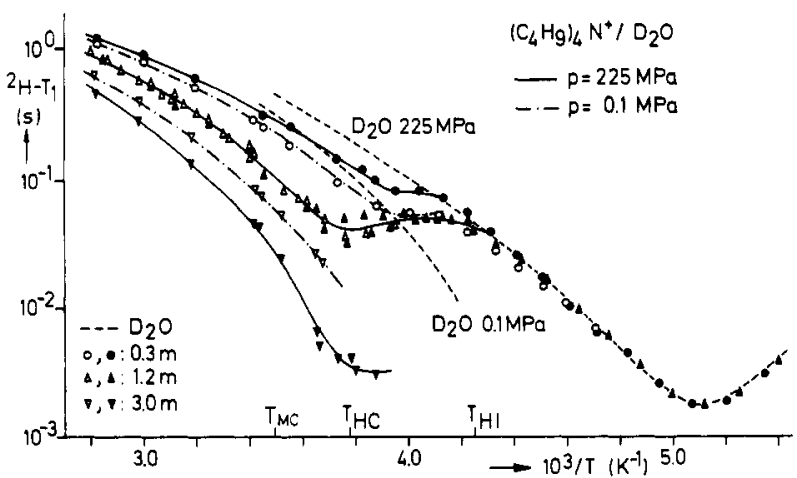

Figure 5. Isobaric temperature dependence of the water ${ }^{2} \mathrm{H} T_{1}$ of undercooled aqueous tetrabutylammonium bromide solutions at the concentrations indicated. Filled symbols represent high-pressure data $(p=225 \mathrm{MPa})$, and open symbols give ambient-pressure $(p=0.1 \mathrm{MPa})$ results. Broken curves give corresponding results of pure $\mathrm{D}_{2} \mathrm{O}, 2,54 T_{\mathrm{MC}}$ $=$ melting temperature of clathrate at $p=0.1 \mathrm{MPa} \cdot{ }^{19} T_{\mathrm{HC}}=$ homogeneous nucleation temperature of clathrate at $p=0.1 \mathrm{MPa} .{ }^{19} T_{\mathrm{HI}}=$ homogeneous nucleation temperature of ice Ih at $p=0.1 \mathrm{MPa} .^{19}$

\section{Data Evaluation}

Hydration Water Relaxation. Contrary to coulombic hydration, the local structure of the hydration shell is less well defined in case of hydrophobic hydration of apolar cations. The structures involved are frequently suggested to be clathratelike and hence should contain many five-membered rings. Water molecules would then be oriented tangentially;i.e., their hydrogen and oxygen atoms are located on average at about the same distance from the ion center. In this way they can maximize their H-bonding requirements. These rather restricted local geometries of the transient $\mathrm{H}$-bond network must reduce the phase space of the hydration water molecules considerably and hence slow down their rotational mobility. Even the clathrate hydrates which the higher members of the $\mathrm{R}_{4} \mathrm{~N}^{+}$series form, however, are characterized by wide ranges of $\mathrm{O}-\mathrm{O}$ distances and $\mathrm{O}-\mathrm{O}-\mathrm{O}$ angles which might be reflected in a corresponding distribution of rotational correlation times. ${ }^{38}$ Therefore, the experimental relaxation rates $R_{1}(T, p, c)$ as a function of temperature at constant pressure have been calculated by eqs 11 and 18 assuming a CD distribution of rotational correlation times. As the deuterium relaxation rates could not be followed into the dispersion regime in case of dissolved $\mathrm{Me}_{4} \mathrm{~N}^{+}$ions, only average correlation times $\langle\tau\rangle$ could be determined corresponding to $\beta=1$ in eq 17 .

The effect of the bromide anions upon the dynamics of the transient $\mathrm{H}$-bond network may be neglected to good approximation. Recent systematic investigations of undercooled aqueous solutions of alkali metal and alkali earth halides ${ }^{1}$ showed a marginal effect of the halide anions (except the $\mathrm{F}^{-}$anion) upon the average mobility of water molecules in these systems. Though neutron scattering experiments and computer simulation studies showed a preferred orientation of the water molecules solvating halide anions, the mean residence time of the former in the solvation shell of the latter seems to be too short for the bromide anions to impose any significant motional constraint upon the water molecules in their solvation shell.

According to the non-Arrhenius temperature dependence of the relaxation rates in the fast-motions limit $\left(R_{1} \approx\langle\tau\rangle, \omega \tau \ll\right.$ 1 ), the average correlation times $(\tau)$ (eq 17) have been assumed to follow a VTF law ${ }^{39-41}$

$$
\langle\tau\rangle=\beta \tau_{0}(T)=\beta \tau_{0} \exp \left[B(p) /\left(T-T_{0}(p, R)\right)\right]
$$

with $T_{0}(p, R)$ a kinetic localization temperature signifying the onset of a motional arrest of the water molecules in the undercooled phase. The empirical VTF law has been found earlier to represent rather well the isobaric temperature dependence of dynamic response functions in a strongly disordered $\mathrm{H}$-bond network of undercooled water and undercooled electrolyte solutions. ${ }^{2}$ The ideal glass transition temperatures $T_{0}$ have been determined in

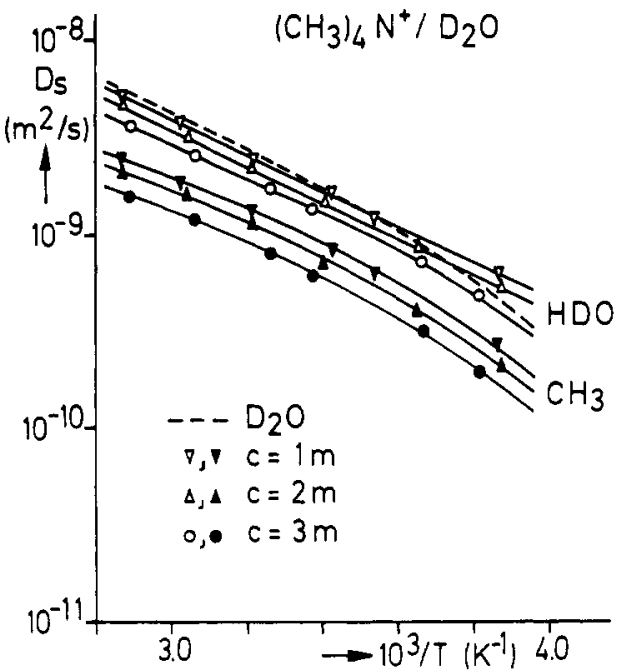

Figure 6. Temperature dependence of the self-diffusion coefficients of the water molecules (open symbols) and the tetramethylammonium cations (filled symbols) at the concentrations indicated. The broken curve represents corresponding self-diffusion coefficients of pure $\mathrm{D}_{2} \mathrm{O} ;{ }^{53}$ full lines represent best fit curves according to a VTF law with the parameters as given in Table $\mathrm{I}$.

TABLE I: Model Parameters To Reproduce the Isobaric Temperature Dependence of the Water Deuteron Spin-Lattice Relaxation Time ( $\left.{ }^{2} \mathrm{H} T_{1}\right)$ and the Self-Diffusion Coefficient $D_{3}$ in Tetramethylammonium Bromide/ $D_{2} O$ Solutions According to the CD Model

(a) Self-Diffusion Coefficients of the Water Molecules $\left(D_{w}\right)$ and the Cations $\left(D_{+}\right)$

\begin{tabular}{|c|c|c|c|c|c|}
\hline \multicolumn{6}{|c|}{$c\left(\mathrm{~mol} \mathrm{salt} / 1 \mathrm{~kg} \mathrm{D} \mathrm{D}_{2} \mathrm{O}\right)=1.0$} \\
\hline $\begin{array}{l}p(\mathrm{MPa}) \\
T_{\mathrm{o}}(\mathrm{K}) \\
D_{\mathrm{w}, 0}\left(10^{8} \mathrm{~m}^{2} / \mathrm{s}\right) \\
D_{+, 0}\left(10^{8} \mathrm{~m}^{2} / \mathrm{s}\right) \\
B_{w}(\mathrm{~K}) \\
B_{+}(\mathrm{K})\end{array}$ & $\begin{array}{l}0.1 \\
143 \\
8.8 \\
4.0 \\
585 \\
586\end{array}$ & $\begin{array}{l}5 \\
134 \\
8.8 \\
4.0 \\
620 \\
611\end{array}$ & $\begin{array}{l}50 \\
130 \\
8.8 \\
4.0 \\
639 \\
633\end{array}$ & $\begin{array}{l}100 \\
125 \\
8.8 \\
4.1 \\
662 \\
659\end{array}$ & $\begin{array}{l}150 \\
125 \\
8.9 \\
4.1 \\
662 \\
666\end{array}$ \\
\hline $\begin{array}{l}p(\mathrm{MPa}) \\
T_{0}(\mathrm{~K}) \\
D_{\mathrm{w}, 0}\left(10^{8} \mathrm{~m}^{2} / \mathrm{s}\right) \\
D_{+, 0}\left(10^{8} \mathrm{~m}^{2} / \mathrm{s}\right) \\
B_{\mathrm{w}}(\mathrm{K}) \\
B_{+}(\mathrm{K})\end{array}$ & $\begin{array}{c}\text { (mol } \\
0.1 \\
138 \\
9.6 \\
4.4 \\
641 \\
641\end{array}$ & $\begin{array}{c}1 \mathrm{~kg} \mathrm{D} \\
5 \\
136 \\
9.6 \\
4.4 \\
642 \\
634\end{array}$ & $\begin{array}{l}=2.0 \\
50 \\
134 \\
9.0 \\
4.3 \\
643 \\
642\end{array}$ & $\begin{array}{l}100 \\
131 \\
8.4 \\
4.2 \\
644 \\
655\end{array}$ & $\begin{array}{l}150 \\
128 \\
7.8 \\
4.1 \\
648 \\
676\end{array}$ \\
\hline $\begin{array}{l}p(\mathrm{MPa}) \\
T_{0}(\mathrm{~K}) \\
D_{\mathrm{w}, 0}\left(10^{8} \mathrm{~m}^{2} / \mathrm{s}\right) \\
D_{+, 0}\left(10^{8} \mathrm{~m}^{2} / \mathrm{s}\right) \\
B_{w}(\mathrm{~K}) \\
B_{+}(\mathrm{K})\end{array}$ & $\begin{array}{c}\text { (mol } \\
0.1 \\
134 \\
8.5 \\
4.2 \\
679 \\
695\end{array}$ & $\begin{array}{l}1 \mathrm{~kg} \text { I } \\
5 \\
134 \\
8.5 \\
4.2 \\
667 \\
689\end{array}$ & $\begin{array}{l}=3.0 \\
50 \\
132 \\
7.7 \\
4.0 \\
661 \\
689\end{array}$ & $\begin{array}{l}100 \\
127 \\
6.9 \\
3.8 \\
661 \\
707\end{array}$ & $\begin{array}{l}150 \\
127 \\
6.1 \\
3.5 \\
645 \\
705\end{array}$ \\
\hline
\end{tabular}

(b) Water Deuteron Spin-Lattice Relaxation Times $\left({ }^{2} \mathrm{H} T_{1}\right)$

\begin{tabular}{cccccc}
\hline$c(m)$ & $p(\mathrm{MPa})$ & $\mathrm{QCC}(\mathrm{kHz})$ & $\langle\tau\rangle(\mathrm{ps})$ & $B(\mathrm{~K})$ & $T_{0}(\mathrm{~K})$ \\
\hline 0.0 & 225 & 202 & 0.05 & 687 & \\
1.0 & 0.1 & 202 & 0.07 & 687 & 143 \\
1.0 & 225 & 202 & 0.07 & 687 & 126 \\
2.0 & 0.1 & 202 & 0.07 & 687 & 138 \\
2.0 & 225 & 202 & 0.07 & 687 & 125 \\
3.0 & 0.1 & 202 & 0.08 & 687 & 133 \\
3.0 & 225 & 202 & 0.09 & 687 & 122
\end{tabular}

aqueous $\mathrm{Me}_{4} \mathrm{NBr}$ solutions by adjusting a VTF law to both the average rotational correlation times $\langle\tau\rangle$ and the corresponding experimental self-diffusion coefficient $D_{\mathrm{w}}$ of the water molecules in these solutions (Figure 6). The respective parameters of best fit are compiled in Table $\mathrm{I}$. In aqueous $\mathrm{Pr}_{4} \mathrm{NBr}$ solutions they have been determined in accord with the concentration dependence of the temperature $T_{\min }$ of the $T_{1}$ minima as was discussed earlier in connection with relaxation studies of undercooled solutions of atomic ions. ${ }^{1,2,42}$ The idea behind is simply that $T_{1}$ minima are obtained whenever $\omega_{\mathrm{L}} \tau_{\text {or }}=\omega_{\mathrm{L}} \tau_{0} \exp \left(B /\left(T_{\min }-T_{0}(R)\right)\right)=1$; 
TABLE II: Model Parameters To Reproduce the Isobaric Temperature Dependence of the Water Deuteron Spin-Lattice Relaxation Time ( $\left.{ }^{2} \mathrm{H} T_{1}\right)$ in Tetrapropylammonium Bromide/ $\mathrm{D}_{2} \mathrm{O}$ Solutions According to the CD Model (Eqs 11, 18, and 19)

\begin{tabular}{ccccccc}
\hline$c(m)$ & $p(\mathrm{MPa})$ & $\mathrm{QCC}(\mathrm{kHz})$ & $\tau_{0}(\mathrm{ps})$ & $B(\mathrm{~K})$ & $T_{0}(\mathrm{~K})$ & $\beta_{\mathrm{CD}}$ \\
\hline 0.3 & 0.1 & 202 & 0.06 & 687 & 149 & 0.81 \\
0.3 & 225 & 202 & 0.09 & 687 & 133 & 0.81 \\
1.4 & 0.1 & 280 & 1.12 & 687 & 166 & 0.16 \\
1.4 & 225 & 280 & 1.43 & 687 & 152 & 0.16 \\
3.0 & 0.1 & 280 & 1.14 & 687 & 173 & 0.16 \\
3.0 & 225 & 280 & 1.87 & 687 & 170 & 0.16 \\
5.0 & 0.1 & 280 & 1.21 & 687 & 177 & 0.17 \\
5.0 & 225 & 280 & 1.73 & 687 & 185 & 0.17
\end{tabular}

hence, $T_{\min }-T_{0}=$ constant irrespective of the composition $R$ of the solution. From a modified Arrhenius diagram $\ln T_{1}\left({ }^{2} \mathrm{H}\right)$ vs $\left(T-T_{0}\right)^{-1}$, the slope parameter $B$ was found not to depend on the composition $R$ of the solutions; therefore, its corresponding pure water value $(B(p=225 \mathrm{MPa})=687.6 \mathrm{~K})$ has been used. The preexponential factor $\tau_{0}$ in eq 19 and the exponent $\beta$ were adjusted in a nonlinear least-squares fit. Furthermore, in concentrated $\mathrm{Pr}_{4} \mathrm{NBr}$ solutions the QCC had to be increased to account for an increase of the value of the relaxation times in the region of the $T_{1}$ minimum. All best fit parameters are compiled in Table II. In addition to the distribution of relaxation times model, the spin-lattice relaxation rates of the $\mathrm{Pr}_{4} \mathrm{~N}^{+} / \mathrm{D}_{2} \mathrm{O}$ systems have been fitted to the two-mode approximation (eq 14) also. As the broad asymmetric relaxation rate spectra indicate, a single motional mode is not sufficient to reproduce the shape of these curves. The simplest approximation then is to envisage two independent motional modes representing slow cooperative overall tumbling motions and exchange processes of hydration water molecules and faster local anisotopic reorientations within the hydration sphere. These overall tumbling and exchange modes must be highly cooperative. Such motions are generally characterized by a strong non-Arrhenius temperature dependence. In contrast, local anisotropic reorientations should be thermally activated and exhibit an Arrhenius temperature dependence. The cooperative overall tumbling mode $\tau_{\text {or }}$ dominates the relaxation in the fastmotions regime as is evident from a non-Arrhenius temperature dependence of the relaxation times. A VTF law has been choosen accordingly

$$
\tau_{\mathrm{or}}=\tau_{0} \exp \left[B(p) /\left(T-T_{0}(p, R)\right)\right]
$$

with $B$ and $T_{0}(p, R)$ taken as before and $\tau_{0}$ considered as an adjustable parameter. The thermally activated local anisotropic mode $\tau_{f}$ should exhibit an Arrhenius dependence in accord with the less strong temperature dependence of the relaxation times in the slow-motions regime where the fast mode provides the main relaxation path. Thus

$$
\tau_{\mathrm{f}}=\left(2 \pi /\left\langle\omega_{\text {lib }}\right\rangle\right) \exp \left(E_{\mathrm{a}} / R T\right)
$$

with $\left\langle\omega_{\text {lib }}\right\rangle$ an average librational frequency of the water molecules. The order parameter $S^{2}$, the preexponential $\tau_{0}$, and the barrier to rotation $E_{\mathrm{a}}$ of the local mode have been adjusted by a leastsquares treatment of the data. The corresponding parameters of best fit are compiled in Table III.

Two-Site Approximation of the Solvent Relaxation. In the case of more dilute solutions ( $m \mathrm{Me}_{4} \mathrm{NBR}$ and $0.3 m \mathrm{Pr}_{4} \mathrm{NBr}$ ), a simple two-site model has been found adequate to calculate the relaxation rate curves from the corresponding hydration water relaxation rates $\left(R_{1}\right)_{\mathrm{h}}(T, p, R)$ and the bulk water relaxation rates $\left(R_{1}\right)_{\mathrm{b}}(T, p, R)$ according to

$$
R_{1}(T, p, R)=\left(N_{\mathrm{h}} / R\right)\left(R_{1}\right)_{\mathrm{h}}+\left(\left(R-N_{\mathrm{h}}\right) / R\right)\left(R_{1}\right)_{\mathrm{b}}
$$

It is essential that both relaxation rates are evaluated at the same reduced temperature $\left(T-T_{0}(p, R)\right)$ with the glass transition temperature $T_{0}(p, R)$ appropriate to the composition $R$ of the solution under investigation. ${ }^{43}$ Relaxation rates at high concentrations $(c>2 m)$ have been calculated from the corresponding hydration water relaxation rate by shifting the glass transition temperature $T_{0}(p, R)$ according to the shift in the temperature of the $T_{1}$ minimum.

\section{Discussion}

Undercooled Aqueous $\mathrm{Me}_{4} \mathrm{NBr}$ Solutions. $\mathrm{Me}_{4} \mathrm{~N}^{+}$ions dissolved in water are sometimes classified as structure breakers ${ }^{44}$ and sometimes said to cause dynamic effects similar to dissolved $\mathrm{Li}^{+}$ ions. ${ }^{6}$ Both of these classifications, drawn from room temperature data, are misleading, however. Concerning the translational and rotational mobility of water molecules as measured directly by self-diffusion coefficients and deuterium spin-lattice relaxation rates in the fast motions regime, it is shown in Figure 2 and Figure 6 that increasing concentrations of dissolved $\mathrm{Me}_{4} \mathrm{~N}^{+}$ions hinder the molecular motions of coordinated water molecules. Though the effect is similar to dissolved $\mathrm{Li}^{+}$ions, it is not due to strong ion-dipole interactions as in the latter case. ${ }^{33,34}$ It has been shown earlier ${ }^{1}$ that the average mobility of water molecules becomes less hindered with decreasing charge density of dissolved atomic ions and finally is facilitated by large monovalent atomic ions like $\mathrm{K}^{+}$and $\mathrm{Cs}^{+} .{ }^{37}$ Though the charge density is further reduced in $\mathrm{Me}_{4} \mathrm{~N}^{+}$ions, they hinder rotational and translational motions of water molecules rather than facilitate them. This slowing down must therefore be a consequence of a reduction in phase space of the coordinated water molecules caused by interactions with the apolar surface of the organic ions as has been correctly stated already by Zeidler and Hertz, ${ }^{45}$ who gave the term "hydration of the second kind" to these phenomena. A structural consequence may be an increased order in terms of better defined relative positions and orientations of the coordinated water molecules as is suggested by Monte Carlo simulations of aqueous solutions of apolar solutes ${ }^{14}$ as well as by molecular theories of the hydrophobic effect. ${ }^{7}$ It should be noted that these considerations relate to a strongly distorted $\mathrm{H}$-bond network caused either by a large thermal energy stored in the motional degrees of freedom or by application of hydrostatic pressure.1,2 Inasmuch as the slowing down of orientational and positional fluctuations of water molecules coordinated to $\mathrm{Me}_{4} \mathrm{~N}^{+}$ions is due to an increased order in the hydration water, $\mathrm{Me}_{4} \mathrm{~N}^{+}$ions hence must be termed structure makers. But this classification depends strongly on the state of the $\mathrm{H}$-bond network as the results in the deeply undercooled phase demonstrate. At low temperatures pure water exhibits a rather well-developed transient $\mathrm{H}$-bond network with largely undisturbed linear $\mathrm{H}$-bonds and an almost undistorted tetrahedral connectivity. ${ }^{3}$ In this state all dissolved atomic ions studied hitherto with NMR disturb the dynamic structure of the transient network and facilitate molecular motions. ${ }^{1,2,42}$ It is interesting to see that much the same is observed with dissolved $\mathrm{Me}_{4} \mathrm{~N}^{+}$ions also. Would these ions induce clathratelike hydration structures with stronger $\mathrm{H}$-bonds compared to bulk water than one might have expected a continued further reduction of the average mobility of the coordinated water molecules with an equally strong temperature dependence of related transport and relaxation coeffcients? Hence, in a state of reduced disorder $\mathrm{Me}_{4} \mathrm{~N}^{+}$-water interactions cannot compete with $\mathrm{H}$-bonding interactions in reducing the phase space of the solvent molecules leading to increased structural correlations within the $\mathrm{H}$-bond network with increasing correlation lengths and increasing time constants of the corresponding fluctuations. Dissolved $\mathrm{Me}_{4} \mathrm{~N}^{+}$ions thus disturb the well-developed $\mathrm{H}$-bond network in cold water and also facilitate molecular motions in line with all atomic ions studied so far. It is remarkable, however, that the latter behavior is observed in undercooled $\mathrm{Me}_{4} \mathrm{~N}^{+}$-water solutions even under high hydrostatic pressure. In this respect they rather resemble large monovalent atomic ions than $\mathrm{Li}^{+}$ions. It is tentative to speculate about similarities in the short-range structure around large monovalent atomic ions and $\mathrm{Me}_{4} \mathrm{~N}^{+}$ions in this connection. Direct structural measurements probing the short-range order in the hydration water have become available only recently. At room temperature neutron diffraction exper- 
TABLE III: Model Parameters To Reproduce the Isobaric Temperature Dependence of the Water Deuteron Spin-Lattice Relaxation Time ( $\left.{ }^{2} \mathrm{H} T_{1}\right)$ in Tetrapropylammonium Bromide/ $\mathrm{D}_{2} \mathrm{O}$ Solutions According to the Two-Mode Approximations (Eq 14)

\begin{tabular}{|c|c|c|c|c|c|c|c|c|}
\hline$c(m)$ & $p(\mathrm{MPa})$ & $\mathrm{QCC}(\mathbf{k H z})$ & $s$ & $\tau_{0}(\mathrm{ps})$ & $B(\mathrm{~K})$ & $T_{0}(\mathrm{~K})$ & $E_{\mathrm{a}}(\mathrm{K})$ & $2 \pi / l i b(p s)$ \\
\hline 0.0 & 225 & 202 & & 0.05 & 687 & & & \\
\hline 0.3 & 0.1 & 185 & 0.41 & 0.13 & 605 & 149 & 2460 & 0.05 \\
\hline 0.3 & 225 & 185 & 0.41 & 0.13 & 687 & 133 & 2460 & 0.05 \\
\hline 1.4 & 0.1 & 185 & 0.41 & 0.13 & 605 & 166 & 2460 & 0.05 \\
\hline 1.4 & 225 & 185 & 0.41 & 0.13 & 687 & 152 & 2460 & 0.05 \\
\hline 3.0 & 0.1 & 185 & 0.35 & 0.19 & 630 & 173 & 2720 & 0.05 \\
\hline 3.0 & 225 & 185 & 0.35 & 0.21 & 687 & 170 & 2620 & 0.05 \\
\hline 5.0 & 0.1 & 185 & 0.39 & 0.23 & 687 & 177 & 2750 & 0.05 \\
\hline 5.0 & 225 & 185 & 0.39 & 0.24 & 687 & 185 & 2800 & 0.05 \\
\hline
\end{tabular}

iments with isotope substitution (NDIS) could show that the hydration of $\mathrm{Me}_{4} \mathrm{~N}^{+}$ions is apolar rather than ionic but failed to show any increased order in the hydration water compared to bulk water. ${ }^{8,9}$ We are currently engaged in NDIS investigations of aqueous $\mathrm{Me}_{4} \mathrm{~N}^{+}$solutions in the undercooled phase. A definitive sharpening of the partial pair correlation functions of water corresponding to better defined short- and medium-range correlations could be observed at low temperatures. 10 Any direct comparison with pure undercooled water is still hampered by a lack of corresponding data of pure water. We are currently performing such experiments also.

In representing the temperature dependence of the orientational correlation times with a VTF law, it has been tacitly assumed that the dynamic structure in these undercooled solutions is correlated with their glass-forming ability. Glass formation of aqueous tetraalkylammonium halide solutions has been investigated over wide ranges of concentration by Kanno et al. ${ }^{16-18}$ These authors measured calorimetric glass transition temperatures $T_{\mathrm{g}}$ which increase with increasing concentration of $\mathrm{Me}_{4} \mathrm{~N}^{+}$ions in the composition range $R=3-7$. For the solutions discussed above a glass transition could not be observed with their cooling rates. The ideal glass transition temperatures $T_{0}(R)$ as deduced from a VTF temperature dependence of the spin-lattice relaxation rates $R_{1}$ and self-diffusion coefficients $D_{w}$ show a negative concentration dependence for these less concentrated solutions. It follows that $T_{0}(R)$ should exhibit a minimum in its concentration dependence as has been observed in other electrolyte solutions also. ${ }^{46} T_{0}(R)$ follows closely the trends observed in aqueous solutions of large atomic ions (see inert of Figure 4) and is in line with the observation that ions which hinder molecular motions of water molecules in a strongly distorted $\mathrm{H}$-bond network exhibit a positive slope $\mathrm{d} T_{0} / \mathrm{d} c>0$ and those which facilitate molecular motions under these conditions have $\mathrm{d} T_{0} / \mathrm{d} c<0 .^{1}$ This is to be expected, however, as a large molecular mobility, and hence short rotational correlation times $\tau_{\text {or }}$ or large self-diffusion coefficients $D_{w}$, corresponds, according to eqs 18,19 , and 20 , at a given temperature to a large distance $\left(T-T_{0}\right)$ from the respective kinetic localization temperature $T_{0}(R)$ where motional arrest is to be expected for vanishingly small cooling rates. ${ }^{47}$

Undercooled Aqueous $\mathrm{Pr}_{4} \mathbf{N B r}$ Solutions. Dissolved $\mathrm{Pr}_{4} \mathrm{~N}^{+}$ions reduced the rotational and translational mobility of water molecules for all concentrations and temperatures studied in a $\mathrm{H}$-bond network strongly disturbed by high hydrostatic pressure. At ambient pressure the rotational mobility is reduced compared to pure water for those water molecules directly coordinated to the apolar cations, i.e., at solution compositions $R \leq 36$. In case of the $0.3 \mathrm{~m}$ solution $(R=167)$ the average solvent mobility is enhanced at low temperatures, suggesting that dissolved $\mathrm{Pr}_{4} \mathrm{~N}^{+}$ ions do not induce clathratelike hydration structures. The latter would be compatible with the network organization of undercooled water ${ }^{3,9}$ and hence should cause an equally dramatic slowing down of the water dynamics. This is not observed, however. Again hydrostatic pressure up to $p=225 \mathrm{MPa}$ has little effect on the deuterium relaxation rates $R_{1}\left({ }^{2} \mathrm{H}\right)$ and self-diffusion coefficients $D_{\mathrm{w}}{ }^{48}$ of the water molecules in the concentration range $0.3 \mathrm{~m} \leq$ $c \leq 5 \mathrm{~m}$. The slowing down of solvent molecular motions with an increasing amount of dissolved $\mathrm{Pr}_{4} \mathrm{~N}^{+}$ions resembles the behavior observed in solutions of strongly hydrating atomic ions
TABLE IV: Model Parameters To Reproduce the Isobaric Temperature Dependence of the Water Deuteron Spin-Lattice Relaxation Time ( ${ }^{2} \mathrm{H} T_{1}$ ) in Pure Undercooled $\mathrm{D}_{2} \mathrm{O}$ and in an Undercooled $11 \mathrm{~m} \mathrm{LiCl} / \mathrm{D}_{2} \mathrm{O}$ Solution According to the $\mathrm{CD}$ Model (Eqs 11, 12, and 18)

\begin{tabular}{lccccc}
\hline & QCC $(\mathrm{kHz})$ & $\tau_{0}(\mathrm{ps})$ & $B(\mathrm{~K})$ & $T_{0}(\mathrm{~K})$ & $\beta_{\mathrm{CB}}$ \\
\hline $\mathrm{D}_{2} \mathrm{O}$ & 222 & 0.036 & 687 & 134 & 0.58 \\
$11 \mathrm{M} \mathrm{LiCl} / \mathrm{D}_{2} \mathrm{O}$ & 264 & 0.082 & 687 & 137 & 0.25
\end{tabular}

under high hydrostatic pressure. But in the case of $\operatorname{Pr}_{4} \mathrm{~N}^{+}$ions the retardation of orientational fluctuations of hydration water molecules must be related to an increasing influence of the apolar surface upon the hydration water dynamics rather than strong ion-dipole interactions as was the case with small atomic ions of a high charge density. Also in $\mathrm{Pr}_{4} \mathrm{~N}^{+}$solutions the spin-lattice relaxation of the hydration water deuterons corresponding to a concentration $c=1.4 \mathrm{~m}$ is less efficient at the rate maximum than in the bulk phase. Accordingly, a less deep relaxation time minimum (Figure 3 ) results as has been found also in case of strongly hydrating atomic ions. ${ }^{49}$

To account for this reduction in relaxation efficiency, the QCC of the hydration water deuterons had to be increased significantly in eq 11 compared to its value in bulk water if the relaxation curves were calculated with the distribution of correlation times model (eq 18). The deuterium QCC of water can be obtained from the relaxation rate maximum, but the result depends on the motional model chosen for the orientational fluctuations of water molecules. ${ }^{42}$ Though the assumption of isotropic orientational fluctuations of water molecules has been found adequate to reproduce the spin-lattice and spin-spin relaxation rate curves of pure water, ${ }^{23,24,26}$ they have been fitted to a CD distribution of correlation times model also to provide suitable reference data of the bulk phase. The corresponding best fit parameters are compiled in Table IV for comparison. A sizable anisotropy corresponding to $\beta=0.58$ results as well as a much larger QCC$\left({ }^{2} \mathrm{H}\right)$ of $\chi=222 \mathrm{kHz}$. The latter turns out to be even further increased in the hydration water of $\mathrm{Pr}_{4} \mathrm{~N}^{+}$ions which is hard to reconcile with the frequently made suggestion of an increasing $\mathrm{H}$-bond strength in case of hydrophobic hydration. It is informative to note that a corresponding increase in the QCC of the hydration water in undercooled $\mathrm{LiCl} / \mathrm{D}_{2} \mathrm{O}$ solutions resulted if the respective deuterium spin-lattice relaxation times were calculated with a CD distribution of correlation times model (see Table IV, Figure 7). This increase is not in accord with quantum mechanical calculations, however, which show that $\mathrm{Li}^{+}$ions will decrease the QCC of the deuterons in coordinated water molecules. ${ }^{50}$ Also, if $\mathrm{H}$-bonds strengthen in the hydration shell of apolar solutes, then the QCC of the hydration water deuterons should decrease according to all theoretical results ${ }^{50,51}$ and also according to a comparison of $\mathrm{QCC}\left({ }^{2} \mathrm{H}\right)$ in ice I and the more disordered higher polymorphs. ${ }^{52}$ Considering these rather unphysical results, the conclusion must be that the $C D$ distribution of correlation times model is inappropriate to represent the rotational dynamics of water molecules in these systems. That the coupling strength had to be increased to account for a decreased relaxation rate maximum indicates that the corresponding amplitudes of the spectral density function $g(\omega \tau)$ are too small in the CD distribution of correlation times model. This effect 

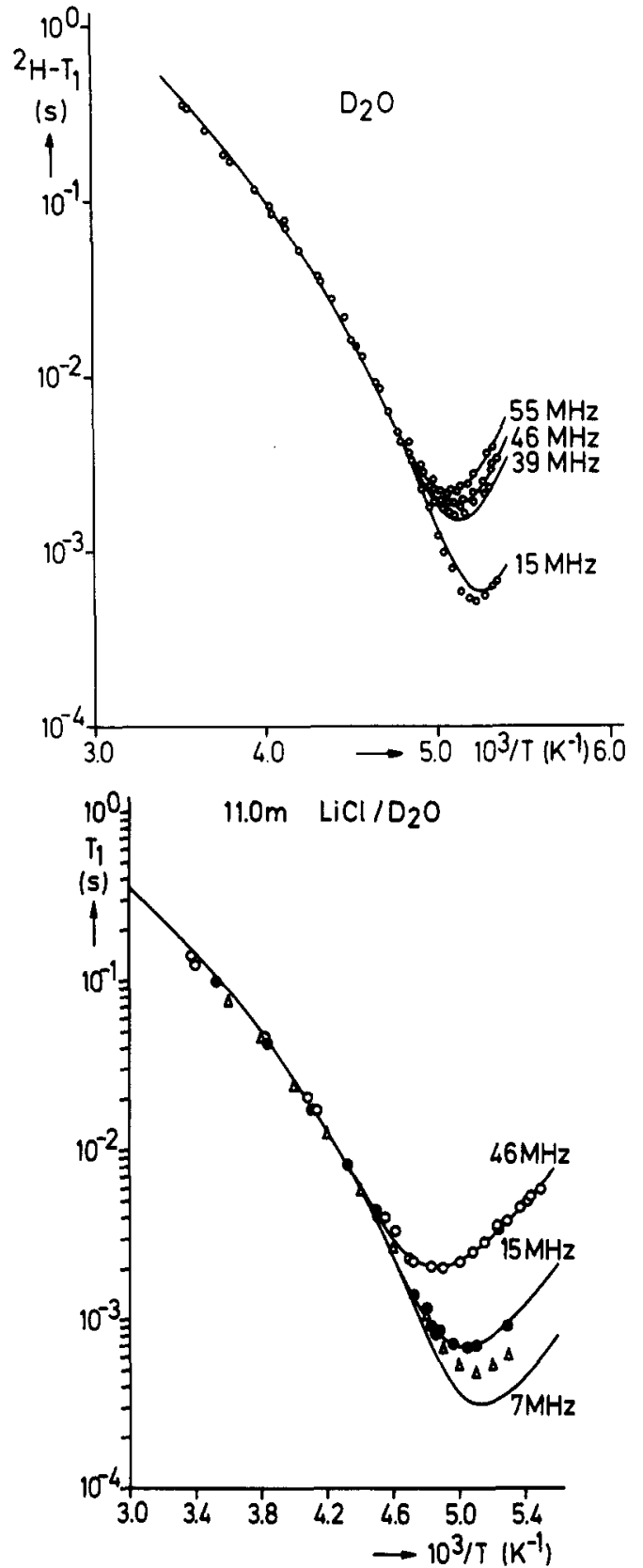

Figure 7. Isobaric $(p=225 \mathrm{MPa})$ temperature dependence of the water ${ }^{2} \mathrm{H} T_{1}$ in undercooled pure $\mathrm{D}_{2} \mathrm{O}^{2}$ and an $11 \mathrm{~m} \mathrm{LiCl} / \mathrm{D}_{2} \mathrm{O}$ solution ${ }^{34}$ at different Larmor frequencies. The data at $7 \mathrm{MHz}$ have been taken from ref 55. Full lines represent best fit curves according to a $C D$ distribution of correlation times model with the parameters given in Table IV.

obviously increases with the asymmetry of the distribution and hence with decreasing $\beta_{\mathrm{CD}}$.

In applying the two-model approximation to calculate the relaxation rates, it has been attempted first to determine the correlation time $\tau_{\text {or }}$ of the slow overall tumbling and exchange modes via

$$
\begin{aligned}
\tau_{\mathrm{s}}(T) & =(2 / 9)\left({R_{+}}^{2} / D_{+}(T)\right) \\
& =(2 / 9)\left({R_{+}}^{2} / D_{0}\right) \exp \left(B(p) /\left(T-T_{0}(p, R)\right)\right.
\end{aligned}
$$

with $R_{+}=5.9 \AA$ the radius of the hydrated cations and $D_{+}$the cationic self-diffusion coefficient. ${ }^{48}$ The respective correlation times turned out to be too long by about an order of magnitude, signifying that the hydration water molecules do not reorient with the cation over a time span given by $\tau_{\mathrm{s}}$ as calculated with eq 23. Hence, the exchange lifetime $\tau_{\mathrm{ex}}$ shortens the effective overall tumbling correlation time $\tau_{\text {or }}$ considerably according to the relation $1 / \tau_{\mathrm{or}}=1 / \tau_{\mathrm{s}}+1 / \tau_{\mathrm{ex}}$. The conclusion must be that the hydration shell around the apolar ions is a highly transient structure with a mean residence time $\tau_{\text {ex }}$ of the hydration water molecules about an order of magnitude shorter than the correlation times $\tau_{\mathrm{s}}$ appropriate to a stable, long-lived hydration shell.

The general order parameters $S^{2}$ indicate a rather substantial spatial restriction of the local anisotropic mode $\tau_{f}$. It turns out to severely influence the shape of the relaxation time curve $T_{1}(T)$ in the slow-motions regime $\left(\omega_{L} \tau_{\text {or }}>1\right)$ where only few data points could be obtained. Hence, these correlation times and the corresponding rotational barriers $E_{\mathrm{a}}$ could not be determined very reliably. In the dispersion region the correlation time $\tau_{\mathrm{f}}$ of the local mode is roughly a factor of 2 shorter than the effective overall tumbling correlation time $\tau_{\text {or }}$ at $T=T_{\min }$, but $\tau_{\mathrm{f}}$ is already shorter by more than 2 orders of magnitude at the lowest temperatures reached. Also in the two-mode approximation the reduction in relaxation efficiency at the rate maximum is solely due to the fast local anisotropic mode providing the main relaxation path at low temperatures. Hence, the coupling strength $\langle\chi\rangle^{2} \mathrm{QLV}$ of the relevant spin-lattice interaction had not to be increased to account for the less efficient relaxation of the hydration water deuterons.

The relaxation rate curves $R_{1}(T, p, c)$ further show that the dynamic structure of the hydration water must be intimately related with the known glass-forming ability of these solutions. ${ }^{16-18}$ The concentration dependence of the ideal glass transition temperature $T_{0}$, as deduced from the temperature $T_{\min }(c)$ of the relaxation time minima $T_{1}\left(T_{\min }\right)$, closely follows the composition dependence of the calorimetric glass transition temperature $T_{\mathrm{g}}(c)$. In this respect it is similar to solutions of strongly hydrating ions which also reduce the rotational mobility of hydration water molecules significantly and increase the corresponding glass transition temperatures where diffusive motions cease to occur. The structural implications of these dynamic features have not yet been explored.

Undercooled Aqueous Bu, $\mathrm{NBr}$ Solutions. Undercooled $\mathrm{Bu}_{4} \mathrm{~N}^{+}$ solutions show the most complex dynamic behavior of all three systems reported in this study. At ambient temperature orientational fluctuations of hydration water molecules are as strongly hindered as with dissolved $\mathrm{Pr}_{4} \mathrm{~N}^{+}$ions. ${ }^{45}$ Their temperature dependence is slightly stronger and seems to parallel the strong power law temperature dependence of these motions in undercooled pure water at ambient pressure. ${ }^{4}$ This is in line with the observation that aqueous $\mathrm{Bu}_{4} \mathrm{NBr}$ solutions do not form a glass but instead form clathrate hydrates rather easily. ${ }^{38}$ The strong temperature dependence of the ionic conductivity in these undercooled solutions has been shown to be closely related to their clathrate forming tendency also. ${ }^{19}$ Our data of the rotational mobility of the water molecules corroborate these observations (Figure 5) and correlate well with the phase behavior in these solutions. ${ }^{19}$ The relaxation times of the 1.2 and $0.3 \mathrm{~m}$ solution level off at the homogeneous nucleation temperature $T_{\mathrm{HC}}(c)$ of the clathrate hydrate (see the phase diagram in ref 19), remain independent of temperature down to the homogeneous nucleation temperature $T_{\mathrm{HI}}$ of ice, and become identical to the relaxation times of pure water if the latter is strongly disturbed by high hydrostatic pressure. 2,24 As Figure 5 demonstrates, the leveling off of the relaxation times, and hence the rotational mobility, occurs at the higher a temperature the higher the concentration is. Also, this temperature shift becomes weaker with increasing concentration well in accord with the observed concentration dependence of $T_{\mathrm{HC}}(c) .{ }^{19}$ On the other hand, the merging of the relaxation times with the bulk water curve occurs at lower temperatures for the higher concentrations as $T_{\mathrm{HI}}$ seems to be suppressed by all solutes added to undercooled water. ${ }^{3}$

This behavior is certainly due to a low-temperature phase separation where a clathrate hydrate separates from the solution and the excess water is forming a highly distorted $\mathrm{H}$-bond network as it is found in pure water under high hydrostatic pressure. At concentrations higher than the eutectic composition, no mobile water remains below the homogeneous nucleation temperature 
$T_{\mathrm{HC}}$ of the clathrate hydrate; hence, the NMR signal corresponding to the liquid phase disappeared.

Hydrostatic pressure is seen to facilitate molecular motions in dilute solutions $(c=0.3 \mathrm{~m})$ in accord with the known pressure effect on the rotational mobility in pure water. Also, the temperature dependence of the relaxation times becomes flatter at high pressure which is observed in pure water, too. It is further interesting to note that no pressure effect is found in the $1.2 \mathrm{~m}$ solution, corresponding to a very small compressibility of the hydration water. This parallels the pressure dependence of the rotational mobility of hydration water molecules in solutions of atomic ions where any pressure effect disappeared at the respective hydration water concentration. ${ }^{33-37}$ At even higher concentrations $(c=3 \mathrm{~m})$ compression strongly hinders rotational motions of those few water molecules confined to the interface between the organic cations.

\section{Conclusions}

NMR relaxation investigations of undercooled aqueous tetraalkylammonium bromide solutions are reported for the first time. The external variables $T, p, c$ have been varied over a large range in order to probe many different states of the random, transient $\mathrm{H}$-bond network. A comparison with related investigations of undercooled alkali-metal halide solutions allowed the competing influence of coulombic, hydrophobic, and $\mathrm{H}$-bond interactions upon the dynamic structure of the random, transient $\mathrm{H}$-bond network to be studied. Due to interactions of the water molecules with the apolar surface of the dissolved $\mathrm{R}_{\mathbf{4}} \mathrm{N}^{+}$ions, solvent dynamics are slowed down in all solutions at room temperature. There the state of the $\mathrm{H}$-bond network corresponds to weak, strongly distorted $\mathrm{H}$-bonds. At low temperatures, the $\mathrm{H}$-bond network is well developed and dissolved $\mathrm{Me}_{4} \mathrm{~N}^{+}$ions enhance the average rotational mobility of the water molecules even close to the apolar surface. The larger $\mathrm{R}_{4} \mathrm{~N}^{+}$cations, when dissolved in a well-developed $\mathrm{H}$-bond network at low temperatures, cause a strongly enhanced average rotational mobility of water molecules in dilute solutions. This dynamic behavior is not compatible with the frequently made suggestion of clathrate cage formation induced in the hydration water by these apolar ions. At a composition where there is just enough water to fully hydrate these apolar ions, the rotational dynamics of the hydration water molecules are, however, strongly slowed down through interaction with the apolar surface. At still higher concentrations water bridging must become important to further reduce the dynamics of the water molecules. For all compositions the solvent dynamics in undercooled solutions of $\mathrm{Pr}_{4} \mathrm{~N}^{+}$ions are closely related to the glass-forming tendency of these systems. In contrast, solutions with dissolved $\mathrm{Bu}_{4} \mathrm{~N}^{+}$ions show a dynamic behavior which is well correlated with their clathrate-forming tendency. The structural implications of these features of the solvent dynamics in these undercooled solutions of apolar ions are still unknown and should be explored with NDIS experiments.

Acknowledgment. The expert technical help of S. Heyne, R. Knott, D. Niesner, and E. Treml is gratefully acknowledged. Prof. Ludemann is thanked for providing access to the MSL 300 NMR spectrometer. Financial support by the DFG is gratefully acknowledged also.

\section{References and Notes}

(1) Lang, E. W.; Fink, W.; Radkowitsch, H.; Girlich, D. Ber. BunsenGes. Phys. Chem. 1990, 94, 342 .

(2) Lang, E. W.; Lüdemann, H.-D. In Jonas, J., Ed. NMR-Basic Principles and Progress; Springer: Berlin, 1990; Vol. 25, pp 131-187.

(3) Angell, C. A. In Franks, F., Ed. Water-A Comprehensive Treatise; Plenum: New York, 1982; Vol. 7, p 1 ff.

(4) Lang, E. W.; Lüdemann, H.-D. Angew. Chem., Int. Ed. Engl. 1982, 21,315 .

(5) Bradl, S.; Lang, E. W. J. Phys.: Condens. Matter 1990, 2, SA195.

(6) Wen, W.-Y. In Horne, R. A., Ed. Water and Aqueous Solutions; Wiley: New York, 1972, pp 613-661.
(7) Huot, J.-Y.; Jolicoeur, C. In Dogonadze, R., Kalman, E., Kornyshev, A. A., Ulstrup, J., Eds. The Chemical Physics of Solvation; Elsevier: Amsterdam, 1985; pp 417-471.

(8) Finney, J.; Turner, J. Faraday Discuss. Chem. 4Soc. 1988, 85, 125.

(9) Turner, J.; Soper, A. K.; Finney, J. Mol. Phys. 1990, 70, 679; 1992. $77,411,431$.

(10) Turner, J.; Soper, A. K.; Finney, J.; Bradl, S.; Lang, E. W. Proc. $I L L-E S R F$ Workshop on Partial Structure Factors, Grenoble, in press.

(11) Stillinger, F. H. Science 1980, 209, 451.

(12) Frank, H. S.; Wen, W.-Y. Discuss. Faraday Soc. 1957, 24, 133.

(13) Jorgensen, W. L.; Gao, J. J. Phys. Chem. 1986, 90, 2174.

(14) Guillot, B.; Guissani, Y.; Bratos, S. J. Chem. Phys. 1991, 95, 3643. 263.

(15) Geiger, A.; Rahman, A.; Stillinger, F. H. J. Chem. Phys. 1979, 70,

(16) Kanno, H.; Shimada, K.; Katoh, K. Chem. Phys. Lett. 1983, 103, 219.

(17) Kanno, H.; Shimada, K.; Yoshino, K.; Iwamoto, T. Chem. Phys. Lett. 1984, 112, 242.

(18) Kanno, H.; Shimada, K.; Katoh, T. J. Phys. Chem. 1989, 93, 4981. 4163.

(19) Mettananda, L. H. I. U.; Speedy, R. J. J. Phys. Chem. 1984, 88,

(20) Spiess, H. W. In Diehl, P., Fluck, E., Kosfeld, R., Eds. NMR-Basic Principles and Progress; Springer: Berlin, 1978; Vol. 15, p $55 \mathrm{ff}$.

(21) Rose, M. F. Elementary Theory of Angular Momentum; Wiley: New York, 1975.

(22) Sceats, M.; Rice, S. A. In Franks, F., Ed. Water-A Comprehensive Treatise; Plenum: New York, 1982; Vol. 7.

(23) Lang, E. W.; Lüdemann, H.-D. J. Chem. Phys. 1977, 67, 718.

(24) Lang, E. W.; Lüdemann, H.-D.; Pjculell, L. J. Chem. Phys. 1984, 81,3820 .

(25) Lang, E. W.; Lüdemann, H.-D. Ber. Bunsen-Ges. Phys. Chem. 1981, 85,603 .

(26) Lang, E. W.; Girlich, D.; Lüdemann, H.-D.; Piculell, L.; Müller, D. J. Chem. Phys. 1990, 93, 4796.

(27) Cummins, P.; Bacskay, G. B.; Hush, N. S.; Engström, S.; Halle, B. J. Chem. Phys. 1985, 82, 2002.

(28) Halle, B.; Wnnnerström, H. J. Chem. Phys. 1981, 75, 1928.

(29) Lipary, G.; Szabo, A. J. Am. Chem. Soc. 1982, 104, 4546, 4559.

(30) Lang, E. W.; Lüdemann, H.-D. Ber. Bunsen-Ges. Phys. Chem. 1985, $89,508$.

(31) Cole, R. H.; Dawidson, D. W. J. Chem. Phys. 1951, 19, 1484.

(32) Eriksson, P.-O.; Lindblom, G.; Burnell, E. E.; Tiddy, G. J. T. J. Chem. Soc., Faraday Trans. 1 1988, 84, 3129.

(33) Lang, E. W.; Fink, W.; Lüdemann, H.-D. J. Phys. 1984, 9, C7-173.

(34) Lang, E. W.; Prielmeier, F. X. Ber. Bunsen-Ges. Phys. Chem. 1988, $92,717$.

(35) Fink, W.; Lang, E. W. J. Phys. Chem. 1988, 92, 6440. 239.

(36) Fink, W.; Radkowitsch, H.; Lang, E. W. Chem. Phys. 1988, 124,

(37) Fink, W.; Radkowitsch, H.; Lang, E. W. Z. Naturforsch. 1988, 43A, 538.

(38) McMullan, R.; Jeffrey, G. A. J. Chem. Phys. 1959, 31, 1231; 1963, 39,3295 .

(39) Vogel, H. Phys. Z 1921, 22, 645.

(40) Tammann, G.; Hesse, W. Z. Anorg. Chem. 1926, 156, 245.

(41) Fulcher, G. S. J. Am. Ceram. Soc. 1925, 77, 3701.

(42) Lang, E. W.; Lüdemann, H.-D. Prog. NMR Spectrosc., in press.

(43) Boden, N.; Mortimer, M. J. Chem. Soc., Faraday Trans. 2 1978, 74, 353.

(44) Kay, R. L.; Evans, D. F. J. Phys. Chem. 1966, 70, 2325.

(45) Hertz, H. G.; Zeidler, M. D. Ber. Bunsen-Ges. Phys. Chem. 1964, 68,821 .

(46) Hofer, K. In Dore, J., Teixeira, J., Eds. Hydrogen Bonded Liquids; Kluwer: The Netherlands, 1991; NATO ASI Ser. C, Vol. 329

(47) Jāckle, J. Rep. Prog. Phys. 1986, 49, 171.

(48) Bradl, S.; Lang, E. W. Manuscript in preparation.

(49) Lang, E. W.; Fink, W.; Radkowitsch, H. In Dore, J. C., Teixeira, J., Eds. Hydrogen Bonded Liquids; Kluwer: The Netherlands, 1991; NATO ASI Ser. C, Vol. 329, p $393 \mathrm{ff}$.

(50) Butler, L. G.; Brown, Th. L. J. Am. Chem. Soc. 1981, 103, 6541.

(51) Berglund, B.; Lindgren, J.; Tegenfeldt, J. J. Mol. Struct. 1978, 43, 179.

(52) Lucken, E. A.C. Nuclear Quadrupole Coupling Constants; Academic Press: London, 1969.

(53) Prielmeier, F. X.; Lang, E. W.; Speedy, R. J.; Lüdemann, H.-D. Ber. Bunsen-Ges. Phys. Chem. 1988, 92, 1111.

(54) Lang, E. W.; Lüdemann, H.-D. Ber. Bunsen-Ges. Phys. Chem. 1980, 84,462 .

(55) Harmon, J. F.; Sutter, E. J. J. Phys. Chem. 1978, 82, 1938. 